\title{
A probiotic complex, rosavin, zinc, and prebiotics ameliorate intestinal inflammation in an acute colitis mouse model
}

Jin-Sil Park ${ }^{1 \dagger}$, JeongWon Choi ${ }^{1 \dagger}$, Ji Ye Kwon ${ }^{1}$, Kyung-Ah Jung ${ }^{2}$, Chul Woo Yang ${ }^{3}$, Sung-Hwan Park ${ }^{1,4^{*} \neq}$ and Mi-La Cho ${ }^{1 *} \neq$ (10)

\begin{abstract}
Background: An altered gut microbiota balance is involved in the pathogenesis of inflammatory bowel disease (IBD), and several probiotic strains are used as dietary supplements to improve intestinal health. We evaluated the therapeutic effect of 12 probiotics in combination with prebiotics, rosavin, and zinc in the dextran sodium sulfate (DSS)induced colitis mouse model.

Methods: The probiotic complex or the combination drug was administered orally to mice with DSS-induced colitis, and the body weight, disease activity index, colon length, and histopathological parameters were evaluated. Also, the combination drug was applied to HT-29 epithelial cells, and the expression of monocyte chemoattractant protein 1 (MCP-1) was evaluated by real-time polymerase chain reaction.

Results: Administration of the combination drug attenuated the severity of DSS-induced colitis. Moreover, the combination drug significantly reduced the levels of the proinflammatory cytokines tumor necrosis factor-a, interleukin (IL)-6, IL-1 $\beta$, and IL-17, and significantly increased the levels of Foxp3 and IL-10 in colon sections. Additionally, treatment with the combination drug reduced MCP-1 expression in HT-29 cells. Treatment with the combination drug decreased the levels of a-smooth muscle actin and type I collagen compared with vehicle treatment in mice with DSS-induced colitis.

Conclusion: These results suggest that the combination of a probiotic complex with rosavin, zinc, and prebiotics exerts a therapeutic effect on IBD by modulating production of pro- and anti-inflammatory cytokines and the development of fibrosis.
\end{abstract}

Keywords: Inflammatory bowel disease, Gut microbiota, Probiotics, Fibrosis

\footnotetext{
*Correspondence: rapark@catholic.ac.kr; iammila@catholic.ac.kr ${ }^{\dagger}$ Jin-Sil Park and JeongWon Choi contributed equally to this work ‡Sung-Hwan Park and Mi-La Cho contributed equally to this work

${ }^{1}$ The Rheumatism Research Center, Catholic Research Institute of Medical

Science, College of Medicine, The Catholic University of Korea, 222,

Banpo-daero, Seocho-gu, Seoul 06591, South Korea

${ }^{4}$ Divison of Rheumatology, Department of Internal Medicine, Seoul

St. Mary's Hospital, The Catholic University of Korea, 222 Banpo-Daero,

Seocho-gu, Seoul 137-701, South Korea

Full list of author information is available at the end of the article
} 


\section{Background}

Inflammatory bowel disease (IBD), which comprises Crohn's disease (CD) and ulcerative colitis (UC), is a chronic, progressive, and destructive inflammatory condition of the gastrointestinal tract caused by multiple genetic and environmental factors [1]. The pathogenesis of IBD is related to a breakdown of intestinal homeostasis, which results in uncontrolled immune responses to the gut microbiota by intestinal epithelial cells and immune cells, leading to complications, such as perforating ulcers and fibrosis [2-5]. Intestinal fibrosis is a common complication and a nonspecific feature of IBD. Increased deposition of collagen causes excessive fibrosis, progressive tissue architectural distortion, dysfunctional wound healing, and luminal narrowing $[6,7]$.

The interplay between the microbiota and immune cells is important in the pathogenesis of IBD [8]. Interleukin (IL)-17-producing Th17 cells are involved in mucosal immune responses $[9,10]$. The IL-17 mRNA level is increased in inflamed mucosa from IBD patients, and the disease severity of IBD is correlated with the IL-17 level in peripheral blood mononuclear cells from UC patients $[11,12]$. Furthermore, IL-17 deficiency does not cause colonic inflammation in IBD animal models [13, 14]. T regulatory (Treg) cells play roles that are the opposite of those played by Th17 cells in autoimmune diseases, including in IBD [15]. Treg cells maintain immunecell homeostasis by suppressing the functions of other immune cell types, particularly Th17 cells. IL-10, which is secreted by Treg cells, is essential for intestinal homeostasis; indeed, IL-10 deficiency leads to the spontaneous development of colitis in mice [16].

Probiotics are live microorganisms that benefit host health when administered in adequate amounts [17]. Probiotics promote maintenance of the gut barrier function and modulation of the host immune system; therefore, dietary supplements containing probiotics may be beneficial for IBD [18-20]. Lactobacillus, Bifidobacterium, and Enterococcus strains are commonly used as probiotics [21-23].

A prebiotic is a non-viable food substance that confers a health benefit on the host by promoting selective growth of beneficial bacteria and is associated with modulation of the intestinal microbiota [24]. A synbiotic, a mixture of a probiotic and prebiotic, has the advantages of both probiotics and prebiotics $[25,26]$.

In this study, we combined 12 probiotics with prebiotics, rosavin (extracted from Rhodiola rosea L.), and zinc. The therapeutic effect of this combination drug in mice with dextran sodium sulfate (DSS)-induced colitis was investigated. The combination drug modulated the production of both proinflammatory cytokines (IL-6, IL-1 $\beta$, and IL-17) and an anti-inflammatory cytokine (IL-10) in mice with DSS-induced colitis. Furthermore, the combination drug ameliorated intestinal fibrosis.

\section{Methods \\ Mice}

Eight-week-old male C57BL/6 mice were purchased from Orient Bio Inc. (Seongnam, Korea). The animals were housed under specific pathogen-free conditions at the Institute of Medical Science of the Catholic University of Korea and were maintained under controlled temperature $\left(21-22{ }^{\circ} \mathrm{C}\right)$ and light (12/12-h light/dark cycle) conditions. The mice were fed standard mouse chow and water. All experimental procedures were approved by the Department of Laboratory Animals, Institutional Animal Care and Use Committee (IACUC) of the School of Medicine, the Catholic University of Korea and conformed with all National Institutes of Health (USA) guidelines (Permit number: CUMC 2016-0244-01).

\section{Probiotic complex, prebiotics, rosavin and zinc}

The probiotic complex (Lot\#171121), prebiotics (Chicory fiber, Lot\#RCRRW6ARW6), and rosavin (Lot\#88843) were purchased from CNS Pharm Korea Co., Ltd (Seoul, Korea). The probiotic complex comprises Lactobacillus acidophilus, Lactobacillus casei, Lactobacillus fermentum, Lactobacillus paracasei, Streptococcous thermophilus, Bifidobacterium longum, Bifidobacterium bifidum, Bifidobacterium breve, Lactobacillus rhamnosus, Lactobacillus plantarum, Lactobacillus helveticus, and Lactobacillus salivarius. The probiotic complex was resuspended in saline at $25 \mathrm{mg} / \mathrm{mL}$ and killed by heating at $80^{\circ} \mathrm{C}$ for $30 \mathrm{~min}$. Zinc was purchased from SigmaAldrich (MO, USA, \#205532).

\section{Induction of colitis and drug administration}

C57BL/6 mice were divided into three groups (5 mice in each group) and were group-housed in cage. Groups of mice were administrated 3\% DSS (MP Biomedicals, Santa Ana, CA, USA) in drink water. To measure the amount of DSS consumption, DSS-dissolved drinking water (3\% DSS) was replaced daily and measure the amount left of drinking water. Each mouse drank about $15 \mathrm{~mL}$ of $3 \%$ DSS water during the 4 days. Mice were randomly assigned to three groups ( $\mathrm{n}=5$ /group) as follows: Vehicle-treated mice were administered DSS in drinking water. Probiotics complex-treated mice were administered the probiotics complex $(100 \mathrm{mg} / \mathrm{mouse})$ in drinking water, and combination drug-treated mice were administered the probiotics complex, prebiotics, zinc, and rosavin $(100 \mathrm{mg} /$ mouse; probiotics complex [1.25 mg], prebiotics [15 mg], zinc [3 $\mathrm{mg}$ ], and rosavin [20 mg]) daily beginning 3 days after DSS administration. Body weight and disease activity index (DAI) score were monitored 
daily. Data are representative of two independent experiments with similar results.

\section{Assessment of inflammation}

The severity of colitis was assessed daily by determining the percentage body weight change and DAI. The DAI was calculated as described previously [27]. Body weight loss (score: 0 , none; $1,0-5 \%$; $2,6-10 \%$; $3,11-15 \%$; $4,16-20 \%$; $5,21-25 \%$; and $6,26-30 \%)$, stool consistency (score: 0 , normal stools; 1 , soft stools; and 2, liquid stools), and rectal bleeding (score: 0 , negative fecal occult blood; 1, positive fecal occult blood; and 2, visible rectal bleeding) were assessed daily in each mouse.

\section{Histopathological analysis}

Colon tissue Sections (5- $\mu \mathrm{m}$ thick) were fixed and embedded in $10 \%(\mathrm{v} / \mathrm{v})$ neutral-buffered formalin (Sigma-Aldrich, St. Louis, MO). Histological analysis was performed on H\&E stained colitis tissue and scored by two experimenters in a blinded fashion. Stained sections were examined under a photomicroscope (Olympus, Tokyo, Japan) (magnifications: 100 $\times$ ). The histological scoring system was used for evaluation of the degree of colitis [28]. Loss of epithelium, crypt damage, depletion of goblet cells, and infiltration of inflammatory cells were assessed in these sections. Histological evaluation and scoring of loss of epithelium ( 0 , no loss of epithelium; 1 , $0-5 \%$ loss of epithelium; $2,5-10 \%$ loss of epithelium; and 3 , > $10 \%$ loss of epithelium), crypt damage ( 0 , no damage; $1,0-10 \%$ loss of crypt; $2,10-20 \%$ loss of crypt; $3,>20 \%$ loss of crypt), depletion of goblet cells ( 0 , none; 1 , mild; 2 , moderate; and 3, severe), and infiltration of inflammatory cells ( 0 , none; 1 , mild; 2 , moderate; and 3 , severe) were performed. Total histological score were ranged from 0 to 12 .

\section{Immunohistochemistry}

Sections were treated with $3 \%(\mathrm{v} / \mathrm{v}) \mathrm{H}_{2} \mathrm{O}_{2}$ in methanol to block endogenous peroxidase activity. Immunohistochemistry was performed using the Envision Detection ${ }^{\text {TM }}$ kit (DAKO, Glostrup, Denmark, \#5007). Tissue sections were incubated with primary antibodies against IL-10 (Santa Cruz Biotechnology, Santa Cruz, CA, USA, \#SC1783), Foxp3 (Santa Cruz Biotechnology, Santa Cruz, CA, USA, \#SC-28705), tumor necrosis factor (TNF)- $\alpha$ (Abcam, Cambridge, UK, \#ab6671), IL-1 $\beta$ (Abcam, Cambridge, UK, \#ab9722), IL-17 (Abcam, Cambridge, UK, \#ab79056), IL-6 (Abcam, Cambridge, UK, \#ab7737), $\alpha$-smooth muscle actin ( $\alpha$-SMA) (Abcam, Cambridge, UK, \#ab7817), and type I collagen (Col-I) (Abcam, Cambridge, UK, \#ab6308) for $2 \mathrm{~h}$ at room temperature. Sections were then incubated with a horseradish peroxidase (HRP)-conjugated secondary antibody for $30 \mathrm{~min}$. The final colored products were developed using chromogen diaminobenzidine, and the sections were examined under a photomicroscope (Olympus, Tokyo, Japan). Positive cells were enumerated visually by four individuals, and the mean values were calculated.

\section{Stimulation of HT-29 cells}

HT-29 cells were seeded in 24-well plates at an initial density of $5 \times 10^{4}$ cells per well and allowed to adhere for $12 \mathrm{~h}$. HT-29 cells were maintained in RPMI-1640 supplemented with $10 \%$ fetal bovine serum. After $12 \mathrm{~h}$, the medium was replaced by a fresh one and the cells were pretreated with combination drug for $2 \mathrm{~h}$ and then the cells were incubated in the presence of lipopolysaccharide (LPS; $1 \mu \mathrm{g} / \mathrm{mL}$, Sigma-Aldrich) for 2 days. The supernatants were assayed for monocyte chemoattractant protein 1 (MCP-1) levels.

\section{Enzyme-linked immunosorbent assay}

The levels of MCP-1 in HT-29 cell culture supernatants were measured by sandwich enzyme-linked immunosorbent assay (ELISA; R\&D Systems). Horseradish peroxidase-avidin (R\&D Systems) was used for color development. Absorbance at $405 \mathrm{~nm}$ was measured using an ELISA microplate reader (Molecular Devices, Sunnyvale, CA, USA).

\section{Statistical analysis}

All statistical analyses were performed using GraphPad Prism (version 4 for Windows; GraphPad Software). Normally distributed continuous data were analyzed using the parametric Student's $t$ test. Differences in mean values among groups were subjected to analysis of variance (ANOVA) followed by Bonferroni post hoc test. Data are presented as means \pm standard deviations (SDs). A value of $p<0.05$ (two-tailed) was considered to indicate statistical significance.

\section{Results \\ Effect of the combination drug on DSS-induced colitis}

To investigate the therapeutic effects of probiotics complex and the combination drug, the probiotics complex and the combination drug in drinking water were administered to mice daily after DSS administration. Mice in the vehicle-treated DSS-induced colitis group showed marked weight loss from day 5 as a result of severe colitis (Fig. 1a). However, treatment with the probiotic complex $(\mathrm{p}<0.05)$ or the combination drug ( $p<0.001)$ ameliorated the loss of body weight. The DAI score, which combines weight loss, changes in stool consistency, and bleeding, was significantly lower in the probiotic complex- $(\mathrm{p}<0.05)$ and combination drug-treated groups $(\mathrm{p}<0.001)$ compared to the 


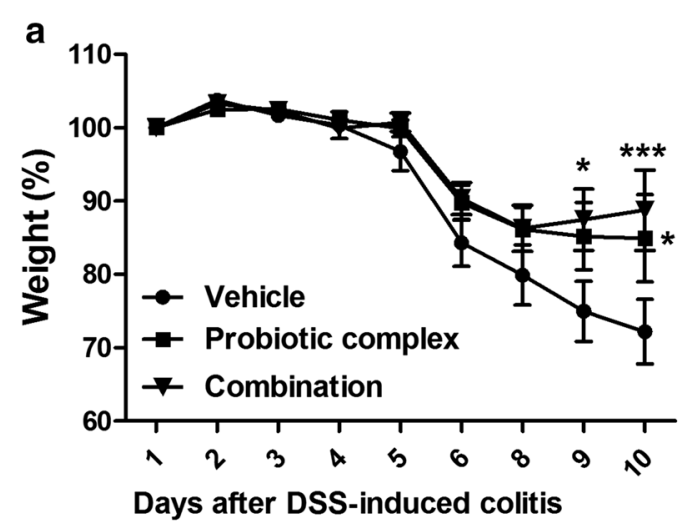

c

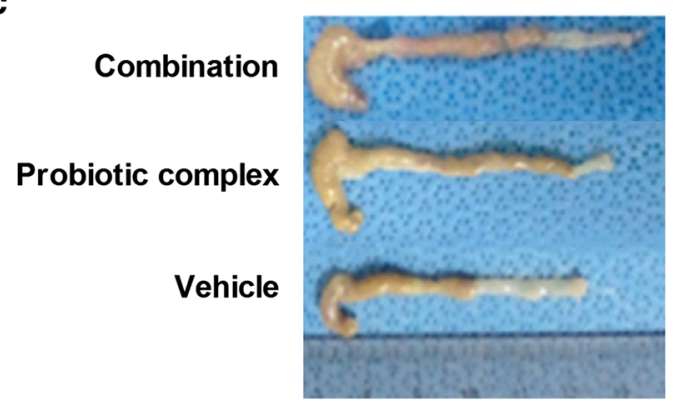

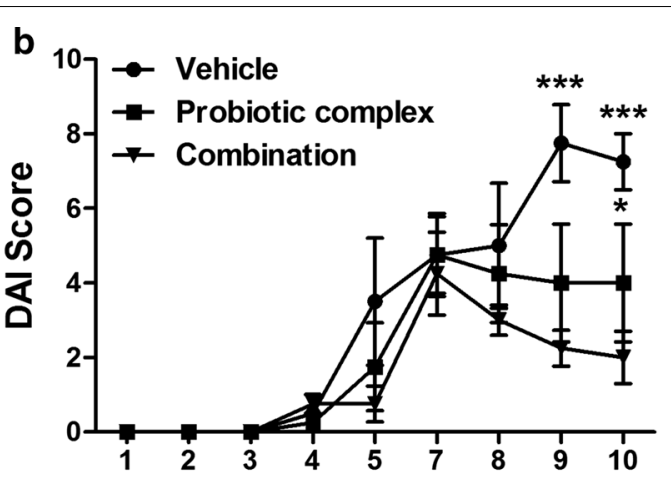

Days after DSS-induced colitis

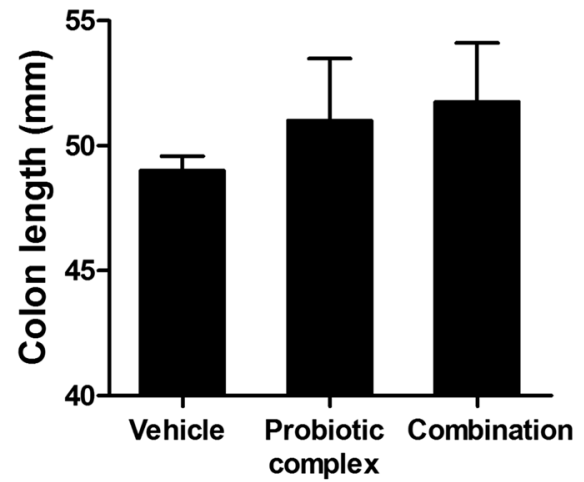

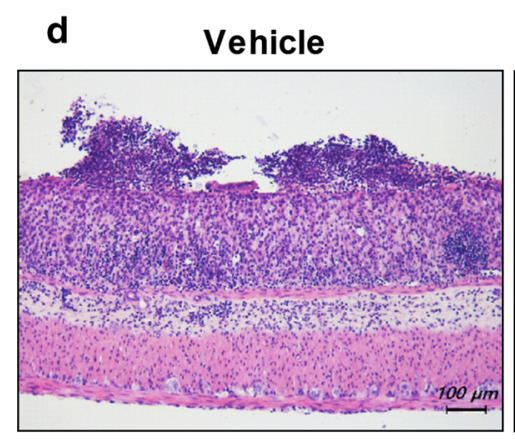
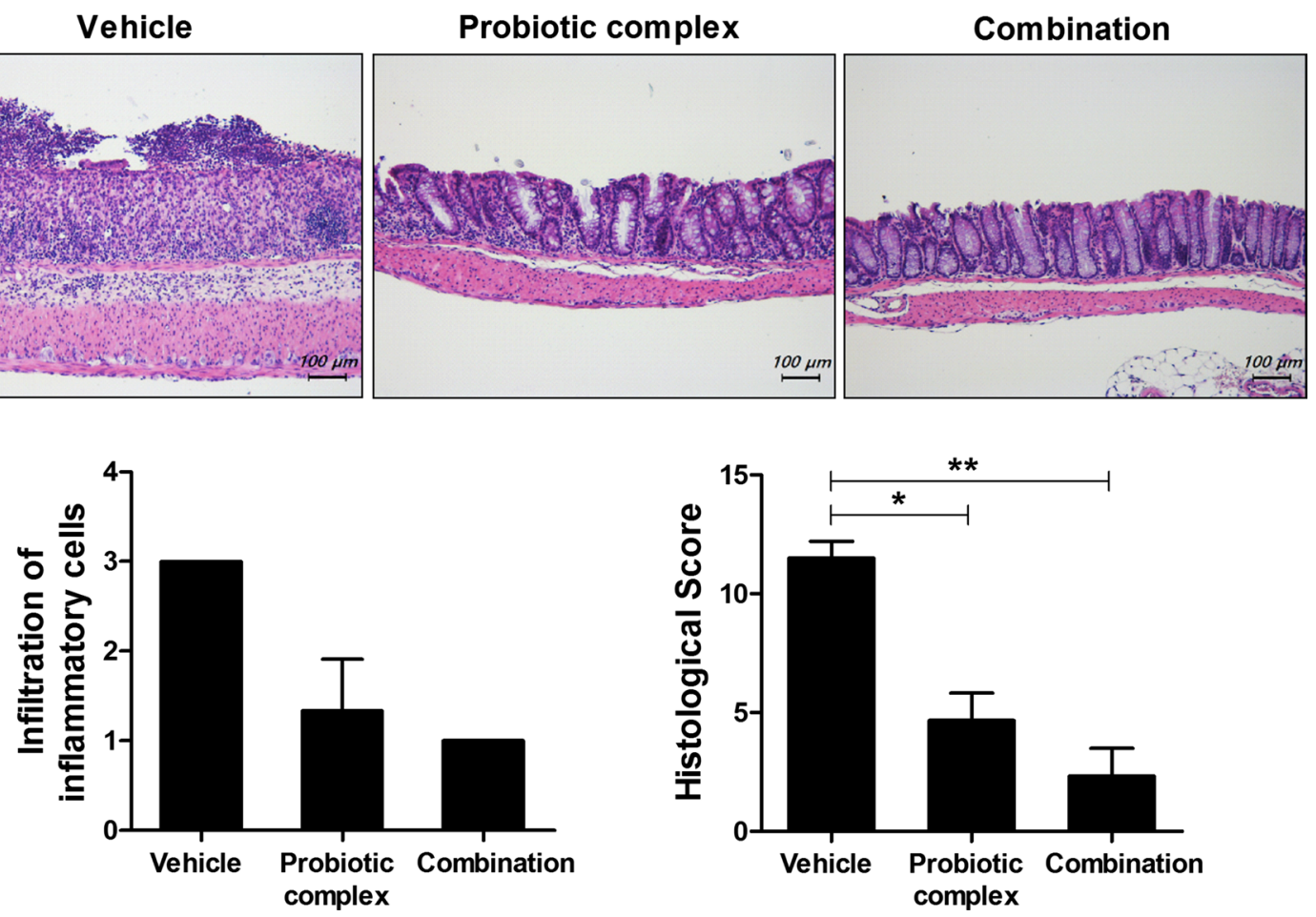
(See figure on previous page.)

Fig. 1 The combination drug ameliorated DSS-induced colitis. C57BL/6 mice were treated orally with 3\% DSS in distilled water ad libitum from days 0 to 4 and thereafter received regular drinking water. On day 3 after DSS administration, the probiotic complex or the combination drug (100 mg/ mouse) was administered orally ( $n=5 /$ group). a Changes in body weight expressed as percentages of body weight on day 0 . b With the exception of day 7, the DAI was monitored daily. Data are means \pm SDs of four independent experiments. c Colon length of mice with DSS-induced chronic colitis on day 10. Macroscopic images of the colon (left panel). Colon length (right panel). $\mathbf{d}$ Representative hematoxylin and eosin-stained images of the colon on day 10. Scale bar, $100 \mu \mathrm{m} .{ }^{*} p<0.05,{ }^{* *} p<0.01,{ }^{* * *} p<0.001$. Vehicle vs. probiotic complex or combination drug treatment

vehicle-treated group (Fig. 1b). Treatment with the probiotic complex or the combination drug prevented the decrease in colon length evident in the vehicle-treated group (Fig. 1c). Microscopic examination of the colon revealed that the probiotic complex and the combination drug restored the mucosal architecture compared with vehicle treatment. The histological score was decreased in the probiotic complex- $(\mathrm{p}<0.05)$ and combination drug-treated groups $(\mathrm{p}<0.01)$ (Fig. 1d). The combination drug exerted a greater therapeutic effect than did the probiotic complex.

\section{Effect of the combination drug on proinflammatory cytokine and chemokine expression}

The effect of the probiotic complex and the combination drug on colitis was assessed by immunostaining
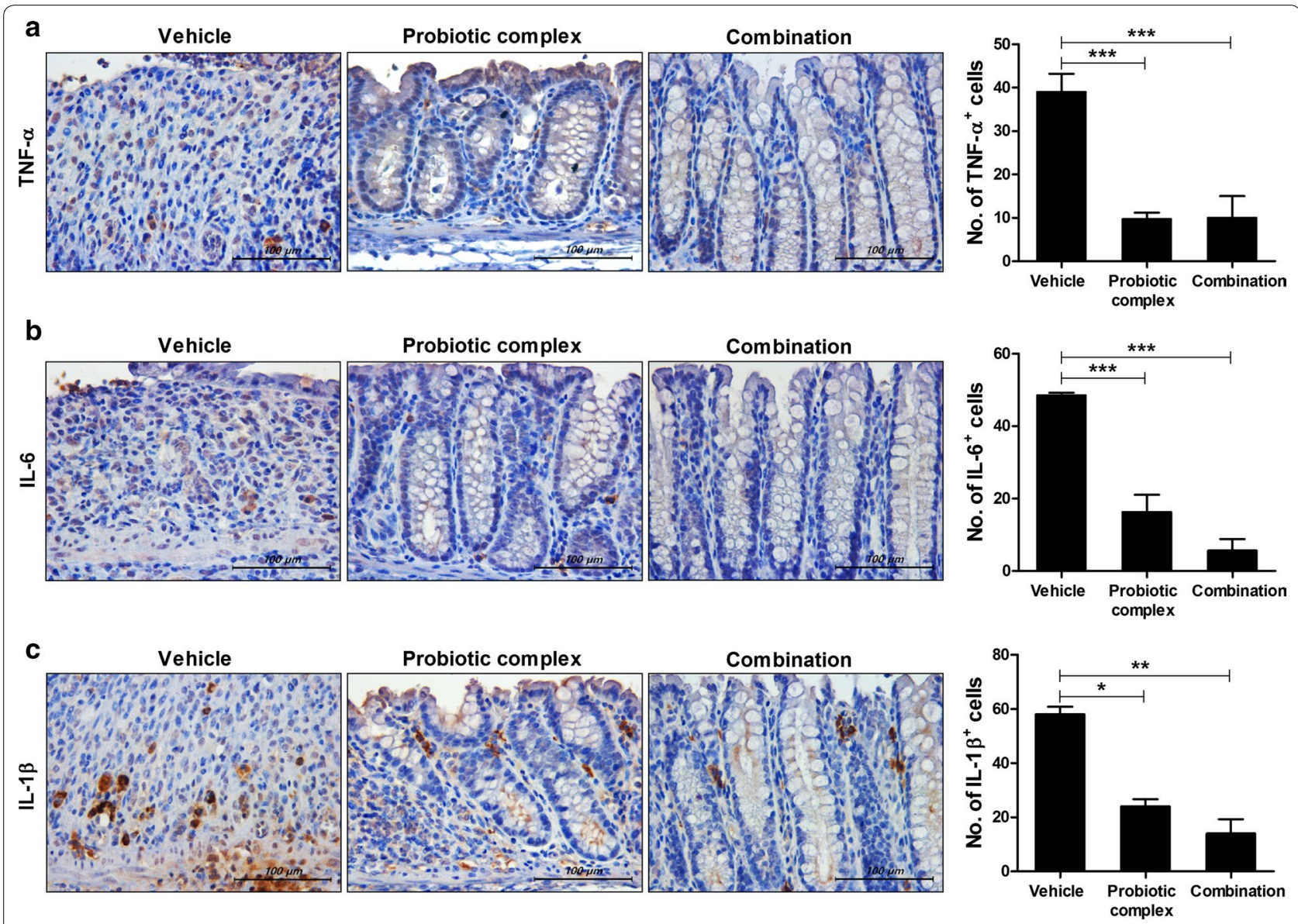

Fig. 2 The combination drug reduced proinflammatory cytokine levels. C57BL/6 mice were treated orally with 3\% DSS in distilled water ad libitum from days 0 to 4 and thereafter received regular drinking water. On day 3 after DSS administration, the probiotic complex or the combination drug (100 mg/mouse) was administered orally ( $n=5 /$ group). On day 10 after DSS administration, colon tissue sections were stained with antibodies against TNF- $a(\mathbf{a})$, IL- $6(\mathbf{b})$, and IL-1 $\beta$ (c). Representative histological features are shown, and the results are presented as mean \pm SD numbers of antibody-positive cells ( $\mathrm{n}=5$ /group). Scale bar, $100 \mu \mathrm{m} .{ }^{*} p<0.05,{ }^{* *} p<0.01,{ }^{* * *} p<0.001$. Vehicle vs. probiotic complex or combination drug treatment 
colon sections for inflammatory cytokines (Fig. 2). Colon tissue from vehicle-treated mice had higher levels of TNF- $\alpha$, IL- 6 , and IL- $1 \beta$ than did colon tissue from mice treated with the probiotic complex or the combination drug; the latter exerted the greatest effect on colitis $(\mathrm{p}<0.001, \mathrm{p}<0.001$, and $\mathrm{p}<0.01$, respectively). To assess the immune modulatory effects of combination drug, the HT-29 cell line, a human colorectal adenocarcinoma cell line, was pretreated with combination drug for $2 \mathrm{~h}$ and then incubated in the presence of LPS for $48 \mathrm{~h}$. The level of MCP-1, which regulates migration and infiltration of monocytes/macrophages [29], in HT-29 cells was decreased by treatment with the combination drug (p < 0.01) (Fig. 3).

\section{Effect of the combination drug on IL-17, Foxp3, and IL-10 levels}

Th17 cells infiltrate the inflamed intestine, where they trigger and amplify inflammation [8]. To investigate the effect of the probiotic complex and the combination drug on IL-17, Foxp3 (a transcription factor for Treg cells [30]), and IL-10 levels, colon sections were subjected to immunohistochemical staining. The colon tissue of mice treated with the probiotic complex or the combination drug exhibited a lower IL-17 level than did the colon tissue of vehicle-treated mice $(\mathrm{p}<0.05)$ (Fig. 4a). In contrast, Foxp3 and IL-10 levels were significantly increased in the colon tissue of mice treated with the combination drug compared to those treated with vehicle $(\mathrm{p}<0.05$ and $\mathrm{p}<0.001$, respectively) (Fig. $4 \mathrm{~b}, \mathrm{c}$ ). Treatment with the probiotic complex also increased the expression of Foxp3 and IL-10, albeit not significantly so.

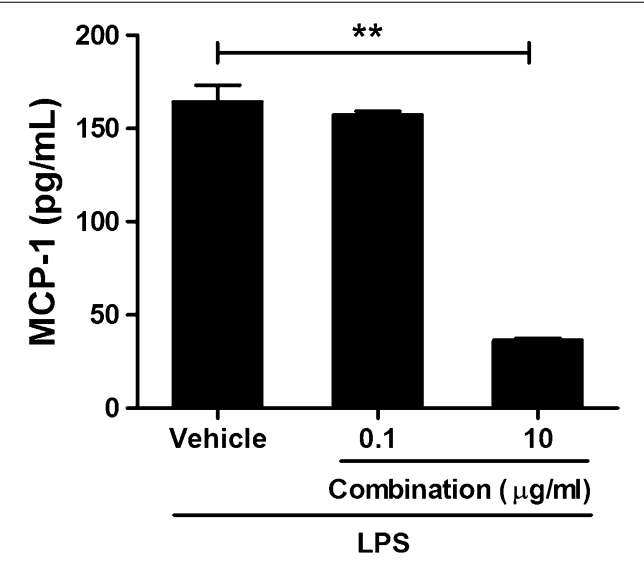

Fig. 3 The combination drug reduced the MCP-1 level in HT-29 cells. $\mathrm{HT}-29$ cells were treated with the combination drug in the presence of LPS $(1 \mu \mathrm{g} / \mathrm{mL})$ for $48 \mathrm{~h}$. MCP-1 mRNA and protein levels were determined by RT-PCR and ELISA, respectively. $p<0.01$ by Student's $t$ test. Vehicle vs. combination drug treatment

\section{Effect of the combination drug on colitis-associated intestinal fibrosis}

To investigate the antifibrotic effects of the probiotic complex and combination drug, colon sections were stained with Masson's trichrome (Fig. 5a). Treatment with the probiotic complex or the combination drug suppressed the area of blue staining in the colonic muscular layer compared with vehicle treatment. To evaluate the effect of the probiotic complex or the combination drug on the number of myofibroblasts in the colonic mucosa and submucosa, immunohistochemical staining for $\alpha$-SMA, a marker of fibroblast differentiation to myofibroblasts, was performed on day 10 . The number of $\alpha$-SMA + myofibroblasts was significantly decreased in the colons of mice treated with the probiotic complex or the combination drug compared with the vehicle-treated mice ( $\mathrm{p}<0.01)$ (Fig. 5b). Furthermore, the number of Col-1+ cells was significantly decreased in the colons of mice treated with the probiotic complex or the combination drug compared with vehicle-treated mice $(\mathrm{p}<0.01)$ (Fig. 5c).

\section{Discussion}

The aim of this study was to elucidate the effect of a combination of a probiotic complex, prebiotics, rosavin, and zinc in a DSS-induced colitis mouse model. Treatment with the combination drug attenuated the severity of colitis; suppressed the body weight loss, reduction in colon length and DAI score; and reduced the histological changes evident in vehicle-treated mice. The combination drug decreased the levels of proinflammatory cytokines and chemokines and increased the levels of Foxp3 and IL-10 in colon tissue. Furthermore, treatment with the combination drug suppressed colitis-associated intestinal fibrosis.

Gut microbiota dysbiosis plays a key role in the pathogenesis of IBD. Probiotics, live microorganisms that benefit host health, are commonly used to control chronic gastrointestinal inflammation in IBD patients. Lactobacillus and Bifidobacterium species are widely used as probiotics [31]. Administration of Lactobacillus plantarum LP ameliorates the severity of colitis in IL-10-knockout mice [32]. Lactobacillus paracasei LS2 isolated from the Korean food, kimchi, reduces colitis disease by decreasing the number of Th1 cells and macrophages in the lamina propria [33]. Treatment with Lactobacillus acidophilus reduces the STAT3 and phosphorylated STAT3 levels in colon tissue from mice with DSS-induced colitis and increases the number of Treg cells among intestinal intraepithelial and lamina propria lymphocytes in a 2,4,6-trinitrobenzene sulfonic acid-induced colitis model $[21,34]$. Treatment with Bifidobacterium longum ameliorates colorectal colitis in rats by altering the methylation 

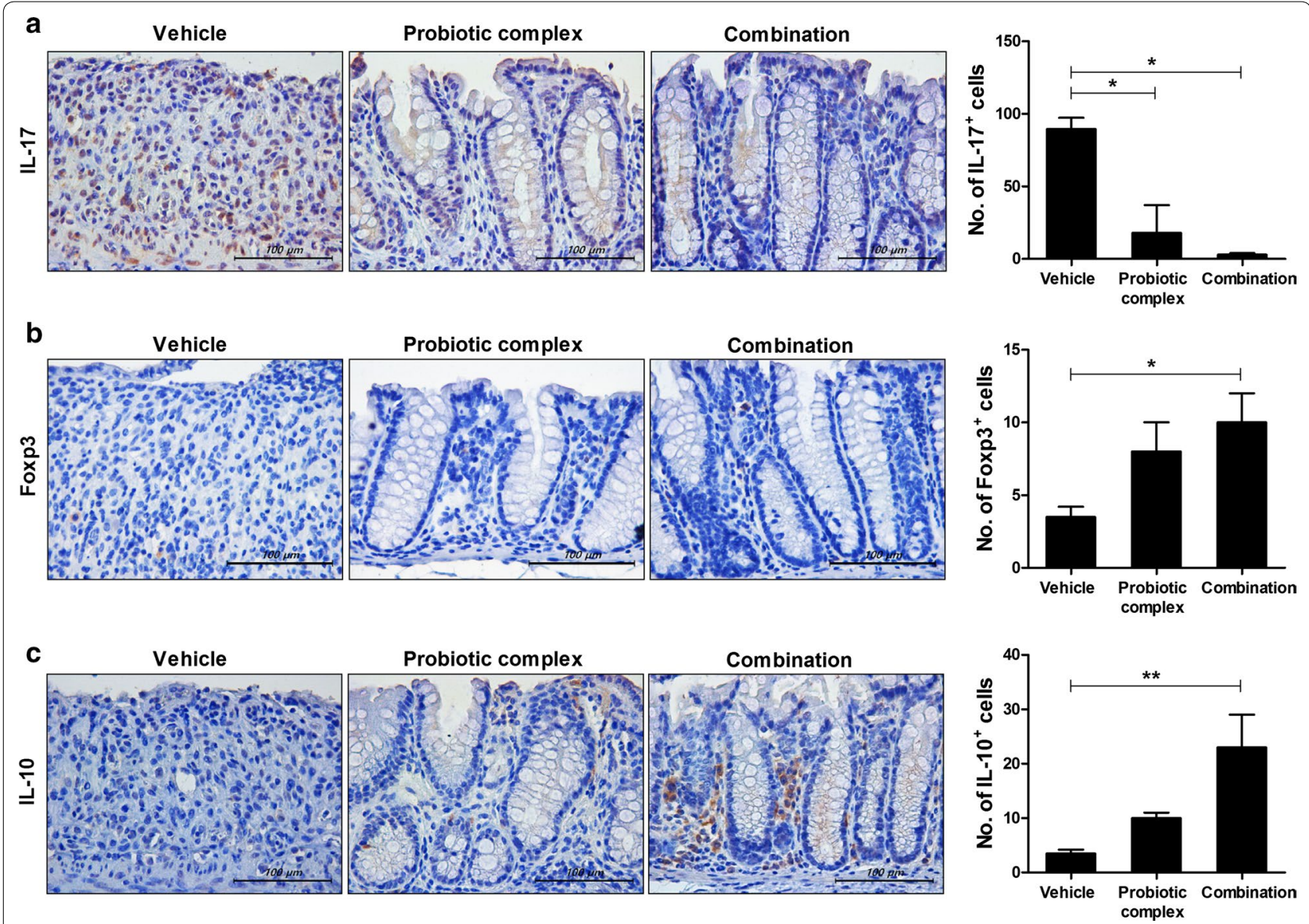

Fig. 4 The combination drug reduces IL-17 and Foxp3 levels. C57BL/6 mice were treated orally with 3\% DSS in distilled water ad libitum from days 0 to 4 and thereafter received regular drinking water. On day 3 after DSS administration, the probiotic complex or the combination drug (100 mg/mouse) was administered orally ( $n=5 / g r o u p)$. On day 10 after DSS administration, colon tissue sections were stained with antibodies against IL-17 (a), Foxp3 (b), and IL-10 (c). Representative histological features are shown, and the results are presented as mean \pm SD numbers of antibody-positive cells $\left(n=5 /\right.$ group). Scale bar, $100 \mu \mathrm{m} .{ }^{*} p<0.05,{ }^{* *} p<0.01$. Vehicle vs. probiotic complex or combination drug treatment

level of the Foxp3 promoter, resulting in an increased number of Treg cells [35]. Moreover, Streptococcus thermophilus suppresses bacterial translocation, which reduces gastrointestinal bleeding and weight loss [36].

Much research has focused on the use of gut microbiota for the treatment of IBD. Treatment with lactobacilli and bifidobacteria promoted recovery of DSS-induced intestinal injury and inflammation in a mouse model of colitis [37]. Lactobacillus casei and Bifidobacterium lactis ameliorated injury to the intestinal mucosa and liver in a 2,4,6-trinitrobenzene sulfonic acid-induced colitis model [38]. Also, treatment with a probiotic combination that included lactobacilli, bifidobacteria, and streptococci reduced the levels of proinflammatory cytokines in colitis [39]. In this study, we evaluated the efficacy of a combination of 12 probiotics (lactobacilli, bifidobacteria, and streptococci) with prebiotics, rosavin, and zinc for the treatment of IBD. Rhodiola rosea is a widely used plant for traditional medicine and it was reported that it has anti-oxidant, neuroprotective, anti-diabetic and anti-inflammatory effects [40-42]. Rosavin is a typical compound of Rhodiola rosea and recent study revealed that it inhibits TNF-related apoptosis-inducing ligand expression via extracellular signal-regulated kinase phosphorylation in T cells [43]. Zinc is absorbed in the small intestine, functions as a cofactor for enzymes, such as alkaline phosphatase, and is essential for growth, immunity, and tissue repair $[44,45]$. Furthermore, zinc involves in the regulation of cell proliferation via affecting metalloenzymes and hormonal signaling. Reduced zinc availability influences survival of animal [46]. Zinc deficiency is commonly seen in IBD patients due to inadequate zinc intake or poor absorption from the gastrointestinal tract $[45,47,48]$. In the present study, oral administration of the combination drug showed therapeutic effects in a DSS-induced colitis model. Notably, the combination 


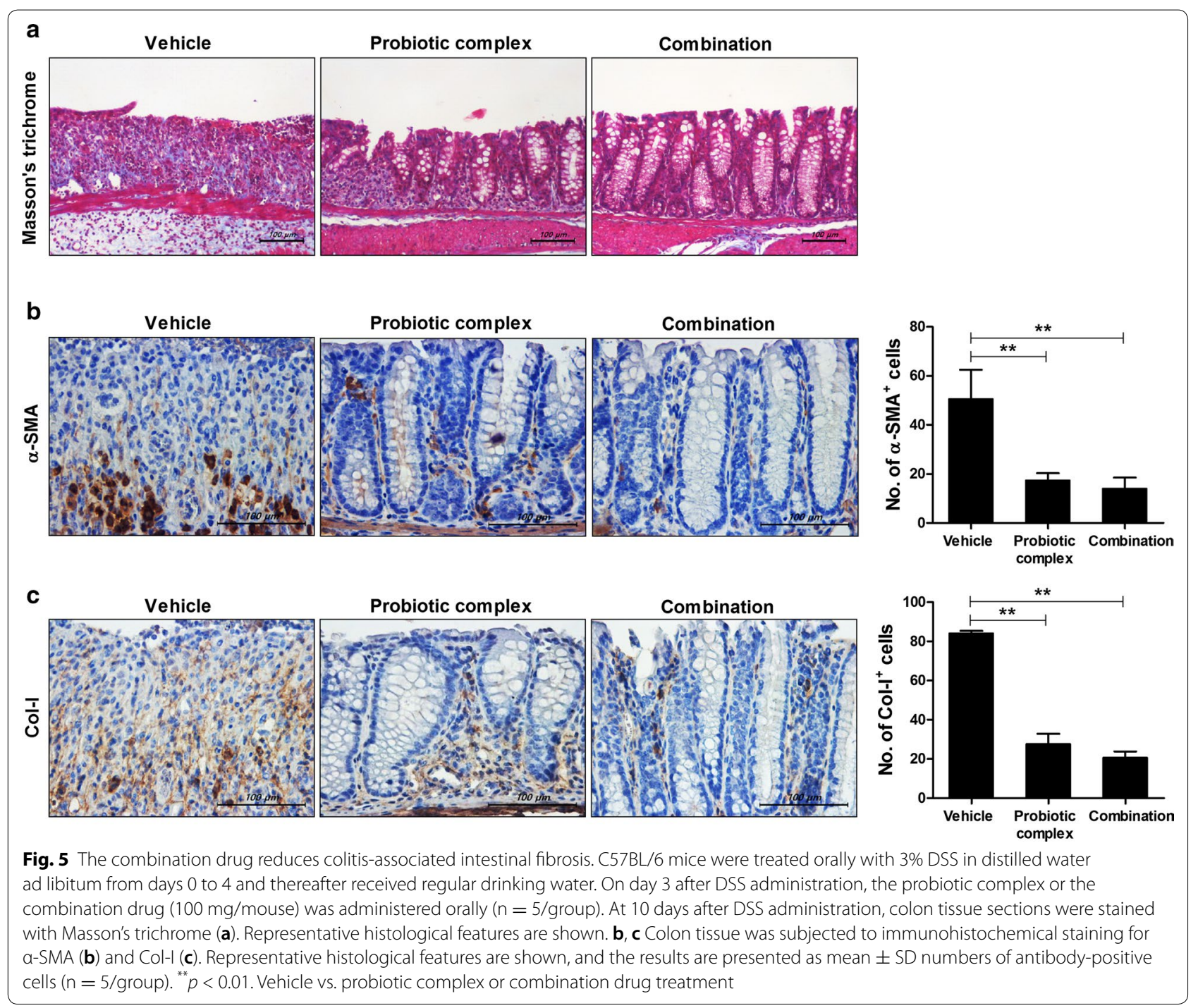

drug resulted in a greater decrease in the levels of proinflammatory cytokines (TNF- $\alpha$, IL- 6 , IL- $1 \beta$, and IL-17) and greater increases in the levels of the anti-inflammatory cytokine IL-10 and transcription factor Foxp3 in colon tissue compared with the probiotic complex alone. Further studies should investigate the mechanisms underlying the effect of the combination drug on intestinal homeostasis.

The combination drug also improved DSS-induced intestinal fibrosis. Fibrosis develops from chronic inflammation and is caused by the accumulation of extracellular matrix components, which causes stricture formation and organ dysfunction [6,7]. Fibrosis is a major and frequent complication in IBD patients, for which no specific therapy is available [49]. The combination drug decreased the $\alpha$-SMA and type I collagen levels compared with vehicle treatment. Recently, there have been attempts to treat fibrosis using cytokine-targeted therapy. IL-10 deficiency accelerates kidney inflammation and fibrosis in the unilateral ureteral obstruction mouse model, and treatment with IL-10 ameliorates lung fibrosis [50, 51]. In contrast, IL-17 exerts pro-fibrotic effects in the lung, liver, and heart [52]. IL-17 deficiency reduces fibrosis in models of skin inflammation, and treatment with IL-17 enhances cardiac fibroblast proliferation and migration in pulmonary fibrosis models [53, 54].

DSS-induced colitis model used in this study is very popular for the IBD research because of its rapidity, simplicity, reproducibility and controllability. However, the limitation of this model is that $\mathrm{T}$ or $\mathrm{B}$ cell responses are not required for the development of colitis unlike human disease [55]. In addition, luminal bacteria play a role in the development of this robust colitis [56]. Therefore, additional studies are needed to determine the complex 
role of combination of a probiotic complex with rosavin, zinc, and prebiotics on the gut-immune axis.

\section{Conclusion}

In conclusion, treatment with the combination of a probiotic complex, prebiotics, rosavin, and zinc attenuated colitis, significantly decreased the levels of proinflammatory cytokines, and significantly increased the levels of Foxp3 and IL-10 in colon tissue. Furthermore, treatment with the combination drug decreased the levels of $\alpha$-SMA and Col-I compared with vehicle treatment. Therefore, the combination of a probiotic complex with rosavin, zinc, and prebiotics may be important therapeutics for IBD by modulating the inflammatory cytokines and fibrosis.

\section{Abbreviations}

IBD: inflammatory bowel disease; CD: Crohn's disease; UC: ulcerative colitis; IL: interleukin; Treg: T regulatory; DAl: disease activity index; TNF: tumor necrosis factor; a-SMA: a-smooth muscle actin; Col-I: type I collagen; LPS: lipopolysaccharide; MCP-1: monocyte chemoattractant protein 1; ELISA: enzyme-linked immunosorbent assay.

\section{Authors' contributions}

JSP, JWC participated in the study design and data interpretation. JSP participated writing the manuscript. JWC, JYK and KAJ carried out the animal experiments, acquired the data and performed the statistical analysis. CWY and SHP participated in the study design and data interpretation. MLC conceived and designed the study, interpreted the data, and made critical revisions of the manuscript for important intellectual content. All authors read and approved the final manuscript.

\section{Author details \\ ${ }^{1}$ The Rheumatism Research Center, Catholic Research Institute of Medical Sci- ence, College of Medicine, The Catholic University of Korea, 222, Banpo-daero, Seocho-gu, Seoul 06591, South Korea. ${ }^{2}$ IMPACT Biotech, Catholic Research Institute of Medical Science, The Catholic University of Korea, Seoul, South Korea. ${ }^{3}$ Division of Nephrology, Department of Internal Medicine, Seoul St. Mary's Hospital, College of Medicine, The Catholic University of Korea, Seoul, South Korea. ${ }^{4}$ Divison of Rheumatology, Department of Internal Medicine, Seoul St. Mary's Hospital, The Catholic University of Korea, 222 Banpo-Daero, Seocho-gu, Seoul 137-701, South Korea.}

\section{Acknowledgements}

None.

\section{Competing interests}

The authors declare that they have no competing interests.

\section{Availability of data and materials}

All data are available in the manuscript or upon request to the authors.

\section{Consent for publication}

Not applicable.

\section{Ethics approval and consent to participate}

All experimental procedures were approved by the Department of Laboratory Animals, Institutional Animal Care and Use Committee (IACUC) of the School of Medicine, the Catholic University of Korea and conformed with all National Institutes of Health (USA) guidelines (Permit Number: CUMC 2016-0244-01).

\section{Funding}

This research was supported by a grant of the Korea Health Technology R\&D Project through the Korea Health Industry Development Institute (KHIDI), funded by the Ministry of Health \& Welfare, Republic of Korea (H115C1062) and by the Bio \& Medical Technology Development Program of the National Research Foundation (NRF) funded by the Ministry of Science, ICT \& Future Planning (NRF-2017M3A9F3041045).

\section{Publisher's Note}

Springer Nature remains neutral with regard to jurisdictional claims in published maps and institutional affiliations.

Received: 14 December 2017 Accepted: 14 February 2018

Published online: 21 February 2018

\section{References}

1. Podolsky DK. Inflammatory bowel disease. N Engl J Med. 2002;347:417-29.

2. Sheil B, Shanahan F, O'Mahony L. Probiotic effects on inflammatory bowel disease. J Nutr. 2007:137:819S-24S.

3. Xavier RJ, Podolsky DK. Unravelling the pathogenesis of inflammatory bowel disease. Nature. 2007:448:427-34.

4. Abraham C, Cho JH. Inflammatory bowel disease. N Engl J Med. 2009:361:2066-78.

5. Van Assche G, Geboes K, Rutgeerts P. Medical therapy for Crohn's disease strictures. Inflamm Bowel Dis. 2004;10:55-60.

6. Speca S, Giusti I, Rieder F, Latella G. Cellular and molecular mechanisms of intestinal fibrosis. World J Gastroenterol. 2012;18:3635-61.

7. Fiocchi C, Lund PK. Themes in fibrosis and gastrointestinal inflammation. Am J Physiol Gastrointest Liver Physiol. 2011;300:G677-83.

8. Galvez J. Role of Th17 cells in the pathogenesis of human IBD. ISRN Inflamm. 2014;2014:928461.

9. Liu ZJ, Yadav PK, Su JL, Wang JS, Fei K. Potential role of Th17 cells in the pathogenesis of inflammatory bowel disease. World J Gastroenterol. 2009;15:5784-8.

10. Owaga E, Hsieh RH, Mugendi B, Masuku S, Shih CK, Chang JS. Th17 cells as potential probiotic therapeutic targets in inflammatory bowel diseases. Int J Mol Sci. 2015;16:20841-58.

11. Sartor RB. Mechanisms of disease: pathogenesis of Crohn's disease and ulcerative colitis. Nat Clin Pract Gastroenterol Hepatol. 2006:3:390-407.

12. Raza A, Shata MT. Letter: pathogenicity of Th17 cells may differ in ulcerative colitis compared with Crohn's disease. Aliment Pharmacol Ther. 2012;36:204 (author reply 205)

13. Ito R, Kita M, Shin-Ya M, Kishida T, Urano A, Takada R, Sakagami J, Imanishi J, Iwakura Y, Okanoue T, et al. Involvement of IL-17A in the pathogenesis of DSS-induced colitis in mice. Biochem Biophys Res Commun. 2008;377:12-6.

14. Zhang Z, Zheng M, Bindas J, Schwarzenberger P, Kolls JK. Critical role of $\mathrm{IL}-17$ receptor signaling in acute TNBS-induced colitis. Inflamm Bowel Dis. 2006;12:382-8

15. Maul J, Loddenkemper C, Mundt P, Berg E, Giese T, Stallmach A, Zeitz M, Duchmann R. Peripheral and intestinal regulatory CD4+ CD25(high) $\mathrm{T}$ cells in inflammatory bowel disease. Gastroenterology. 2005;128:1868-78.

16. Gomes-Santos AC, Moreira TG, Castro-Junior AB, Horta BC, Lemos L, Cruz DN, Guimaraes MA, Cara DC, McCafferty DM, Faria AM. New insights into the immunological changes in IL-10-deficient mice during the course of spontaneous inflammation in the gut mucosa. Clin Dev Immunol. 2012;2012:560817.

17. Bermudez-Brito M, Plaza-Diaz J, Munoz-Quezada S, Gomez-Llorente C, Gil A. Probiotic mechanisms of action. Ann Nutr Metab. 2012;61:160-74.

18. Mortaz E, Adcock IM, Ricciardolo FL, Varahram M, Jamaati H, Velayati AA, Folkerts G, Garssen J. Anti-inflammatory effects of lactobacillus rahmnosus and bifidobacterium breve on cigarette smoke activated human macrophages. PLoS ONE. 2015;10:e0136455.

19. Jones SE, Versalovic J. Probiotic Lactobacillus reuteribiofilms produce antimicrobial and anti-inflammatory factors. BMC Microbiol. 2009;9:35. 
20. Plaza-Diaz J, Gomez-Llorente C, Campana-Martin L, Matencio E, Ortuno I, Martinez-Silla R, Gomez-Gallego C, Periago MJ, Ros G, Chenoll E, et al. Safety and immunomodulatory effects of three probiotic strains isolated from the feces of breast-fed infants in healthy adults: SETOPROB study. PLOS ONE. 2013;8:e78111.

21. Chen L, Zou Y, Peng J, Lu F, Yin Y, Li F, Yang J. Lactobacillus acidophilus suppresses colitis-associated activation of the IL-23/Th17 axis. J Immunol Res. 2015;2015:909514.

22. Gibson GR, Roberfroid MB. Dietary modulation of the human colonic microbiota: introducing the concept of prebiotics. J Nutr. 1995;125:1401-12.

23. Fontana L, Bermudez-Brito M, Plaza-Diaz J, Munoz-Quezada S, Gil A. Sources, isolation, characterisation and evaluation of probiotics. Br J Nutr. 2013;109(Suppl 2):S35-50

24. Gibson GR, Probert HM, Loo JV, Rastall RA, Roberfroid MB. Dietary modulation of the human colonic microbiota: updating the concept of prebiotics. Nutr Res Rev. 2004;17:259-75.

25. Kolida S, Gibson GR. Synbiotics in health and disease. Annu Rev Food Sc Technol. 2011;2:373-93.

26. Sanders ME, Marco ML. Food formats for effective delivery of probiotics. Annu Rev Food Sci Technol. 2010;1:65-85.

27. Suzuki K, Sun X, Nagata M, Kawase T, Yamaguchi H, Sukumaran V, Kawauchi Y, Kawachi H, Nishino T, Watanabe K, et al. Analysis of intestinal fibrosis in chronic colitis in mice induced by dextran sulfate sodium. Pathol Int. 2011;61:228-38.

28. Han ES, Oh JY, Park HJ. Cordyceps militaris extract suppresses dextran sodium sulfate-induced acute colitis in mice and production of inflammatory mediators from macrophages and mast cells. J Ethnopharmacol. 2011:134:703-10

29. Deshmane SL, Kremlev S, Amini S, Sawaya BE. Monocyte chemoattractant protein-1 (MCP-1): an overview. J Interferon Cytokine Res. 2009;29:313-26

30. Zhang $L$, Zhao $Y$. The regulation of Foxp3 expression in regulatory CD4(+)CD25(+)T cells: multiple pathways on the road. J Cell Physiol. 2007:211:590-7.

31. Stanton C, Gardiner G, Meehan H, Collins K, Fitzgerald G, Lynch PB, Ross RP. Market potential for probiotics. Am J Clin Nutr. 2001;73:476S-83S.

32. Xia Y, Chen HQ, Zhang M, Jiang YQ, Hang XM, Qin HL. Effect of Lactobacillus plantarum LP-Onlly on gut flora and colitis in interleukin-10 knockout mice. J Gastroenterol Hepatol. 2011;26:405-11.

33. Park JS, Joe I, Rhee PD, Jeong CS, Jeong G. A lactic acid bacterium isolated from kimchi ameliorates intestinal inflammation in DSS-induced colitis. J Microbiol. 2017;55:304-10

34. Roselli M, Finamore A, Nuccitelli S, Carnevali P, Brigidi P, Vitali B, Nobili F, Rami R, Garaguso I, Mengheri E. Prevention of TNBS-induced colitis by different Lactobacillus and Bifidobacterium strains is associated with an expansion of gammadeltaT and regulatory $T$ cells of intestinal intraepithelial lymphocytes. Inflamm Bowel Dis. 2009;15:1526-36.

35. Zhang M, Zhou L, Zhang S, Yang Y, Xu L, Hua Z, Zou X. Bifidobacterium longum affects the methylation level of forkhead box P3 promoter in 2 , 4, 6-trinitrobenzenesulphonic acid induced colitis in rats. Microb Pathog. 2017;110:426-30.

36. Bailey JR, Vince V, Williams NA, Cogan TA. Streptococcus thermophilus NCIMB 41856 ameliorates signs of colitis in an animal model of inflammatory bowel disease. Benef Microbes. 2017:8:605-14.

37. Toumi R, Soufli I, Rafa H, Belkhelfa M, Biad A, Touil-Boukoffa C. Probiotic bacteria lactobacillus and bifidobacterium attenuate inflammation in dextran sulfate sodium-induced experimental colitis in mice. Int J Immunopathol Pharmacol. 2014;27:615-27.

38. Bellavia M, Rappa F, Lo Bello M, Brecchia G, Tomasello G, Leone A, Spatola G, Uzzo ML, Bonaventura G, David S, et al. Lactobacillus casei and bifidobacterium lactis supplementation reduces tissue damage of intestinal mucosa and liver after 2,4,6-trinitrobenzenesulfonic acid treatment in mice. J Biol Regul Homeost Agents. 2014;28:251-61.

39. Kim MS, Byun JS, Yoon YS, Yum DY, Chung MJ, Lee JC. A probiotic combination attenuates experimental colitis through inhibition of innate cytokine production. Benef Microbes. 2017:8:231-41.

40. Wang M, Luo L, Yao L, Wang C, Jiang K, Liu X, Xu M, Shen N, Guo S, Sun C, Yang Y. Salidroside improves glucose homeostasis in obese mice by repressing inflammation in white adipose tissues and improving leptin sensitivity in hypothalamus. Sci Rep. 2016;6:25399.
41. Chen X, Liu J, Gu X, Ding F. Salidroside attenuates glutamate-induced apoptotic cell death in primary cultured hippocampal neurons of rats. Brain Res. 2008:1238:189-98.

42. Zhao G, Shi A, Fan Z, Du Y. Salidroside inhibits the growth of human breast cancer in vitro and in vivo. Oncol Rep. 2015;33:2553-60.

43. Marchev AS, Dimitrova P, Koycheva IK, Georgiev MI. Altered expression of TRAIL on mouse T cells via ERK phosphorylation by Rhodiola rosea L. and its marker compounds. Food Chem Toxicol. 2017;108:419-28.

44. Turvey SE, Broide DH. Innate immunity. J Allergy Clin Immunol. 2010;125:S24-32.

45. Alkhouri RH, Hashmi H, Baker RD, Gelfond D, Baker SS. Vitamin and mineral status in patients with inflammatory bowel disease. J Pediatr Gastroenterol Nutr. 2013;56:89-92.

46. MacDonald RS. The role of zinc in growth and cell proliferation. J Nutr. 2000;130:1500S-8S

47. Vagianos K, Bector S, McConnell J, Bernstein CN. Nutrition assessment of patients with inflammatory bowel disease. JPEN J Parenter Enteral Nutr. 2007:31:311-9.

48. Ojuawo A, Keith L. The serum concentrations of zinc, copper and selenium in children with inflammatory bowel disease. Cent Afr J Med. 2002:48:116-9.

49. Fichera A, Lovadina S, Rubin M, Cimino F, Hurst RD, Michelassi F. Patterns and operative treatment of recurrent Crohn's disease: a prospective longitudinal study. Surgery. 2006;140:649-54.

50. Louis H, Van Laethem JL, Wu W, Quertinmont E, Degraef C, Van den Berg K, Demols A, Goldman M, Le Moine O, Geerts A, Deviere J. Interleukin-10 controls neutrophilic infiltration, hepatocyte proliferation, and liver fibrosis induced by carbon tetrachloride in mice. Hepatology. 1998;28:1607-15.

51. Jin Y, Liu R, Xie J, Xiong H, He JC, Chen N. Interleukin-10 deficiency aggravates kidney inflammation and fibrosis in the unilateral ureteral obstruction mouse model. Lab Invest. 2013;93:801-11.

52. Liu Y, Zhu H, Su Z, Sun C, Yin J, Yuan H, Sandoghchian S, Jiao Z, Wang S, Xu H. IL-17 contributes to cardiac fibrosis following experimental autoimmune myocarditis by a PKCbeta/Erk1/2/NF-kappaB-dependent signaling pathway. Int Immunol. 2012;24:605-12.

53. Okamoto Y, Hasegawa M, Matsushita T, Hamaguchi Y, Huu DL, I wakura Y, Fujimoto M, Takehara K. Potential roles of interleukin-17A in the development of skin fibrosis in mice. Arthritis Rheum. 2012;64:3726-35.

54. Valente AJ, Yoshida T, Gardner JD, Somanna N, Delafontaine P, Chandrasekar B. Interleukin-17A stimulates cardiac fibroblast proliferation and migration via negative regulation of the dual-specificity phosphatase MKP-1/DUSP-1. Cell Signal. 2012;24:560-8.

55. Chassaing B, Aitken JD, Malleshappa M, Vijay-Kumar M. Dextran sulfate sodium (DSS)-induced colitis in mice. Curr Protoc Immunol. 2014;104:15-25.

56. Yamada M, Ohkusa T, Okayasu I. Occurrence of dysplasia and adenocarcinoma after experimental chronic ulcerative colitis in hamsters induced by dextran sulphate sodium. Gut. 1992;33:1521-7.

\section{Submit your next manuscript to BioMed Central and we will help you at every step:}

- We accept pre-submission inquiries

- Our selector tool helps you to find the most relevant journal

- We provide round the clock customer support

- Convenient online submission

- Thorough peer review

- Inclusion in PubMed and all major indexing services

- Maximum visibility for your research

Submit your manuscript at www.biomedcentral.com/submit 